\title{
Crohn's disease or TB - the perennial question and diagnostic pitfalls
}

\author{
Rudra Krishna Maitra, ${ }^{1}$ Tim Bowling, ${ }^{2}$ Pradhib Venkatesan, ${ }^{3}$ Charles Maxwell-Armstrong ${ }^{1}$
}

${ }^{1}$ Surgery Department, Queens Medical Centre, Nottingham University Hospitals, Nottingham, UK;

${ }^{2}$ Gastroenterology Department, Queens Medical Centre, Nottingham University Hospitals, Nottingham, UK:

${ }^{3}$ Infectious Diseases Department, City Hospital, Nottingham University Hospitals, Nottingham, UK;

Correspondence to Mr Rudra Krishna Maitra, rudra.k.maitra@gmail.com

\begin{abstract}
Summary
A previously healthy 28-year old lady from Saudi Arabia presented with recurrent peri-anal abscesses progressing to fistula-in-ano. These were treated with incision and drainages and with setonisation of the fistula. Multiple biopsy and culture specimens were taken to rule out tuberculosis (TB) or Crohn's disease - all showed granulomatous disease suggestive of either Crohn's or TB, no mycobacteria were grown. MRI scanning also suggested either TB or Crohn's disease. Tuberculin skin test was inconclusive and Quantiferon Gold test was negative. Treatment for Crohn's was started with oral prednisolone - the patient deteriorated and adalimumab (tumour necrosis factor $\alpha$ antagonist) was commenced. With continued deterioration in the absence of intra-abdominal abscesses, a clinical diagnosis of TB was made, Crohn's treatment suspended and quadruple therapy for TB was initiated. The patient rapidly improved and a delayed re-look histological specimen identified an isolated mycobacterium. Subsequent cultures confirmed drug-sensitive TB. The lady is currently well on TB eradication regimen.
\end{abstract}

\section{BACKGROUND}

In the UK, Crohn's disease (CD) has an incidence of 8.3 per 100000 of population and is a common cause of recurrent peri-anal abscesses and fistulation. Tuberculosis (TB) is uncommon in the UK with a prevalence of 15 per 100000 of the population (2009) of which only a small fraction present as intestinal TB; prevalence is significantly higher in developing countries. ${ }^{12}$ Diagnosing TB is difficult with no test providing $100 \%$ sensitivity and specificity. Delayed diagnoses can result in serious morbidity. Extra-pulmonary manifestations of TB accounted for $47 \%$ of cases in the UK (2009), more commonly in foreign-born individuals who account for the majority of cases. ${ }^{2}$ Intestinal TB presents with clinical features resembling $\mathrm{CD}$ and must be distinguished from it.

In the following case, TB was suspected as a differential for $\mathrm{CD}$ from the outset but all tests proved negative for $\mathrm{TB}$. TB was correctly diagnosed after clinical deterioration following initiation of treatment for $\mathrm{CD}$. This case highlights the difficulty of distinguishing intestinal $\mathrm{TB}$ and $\mathrm{CD}$ and the need for constant re-evaluation of the diagnosis in the face of deterioration.

\section{CASE PRESENTATION}

A previously well 28-year-old lady from Saudi Arabia presented with a left-sided peri-anal abscess. She had BCG immunisation as a child and gave no personal, family or contact history of TB. In Saudi Arabia she did not drink any unpasteurised milk. She had a history of a previous peri-anal abscess. She was systemically well. An incision and drainage of the abscess was performed. Microbiology samples grew Escherichia coli and Enterococcus, both sensitive to co-amoxiclav, with which she was treated.

She returned 5 months later with a slow healing wound and discharge from the opposite side.

\section{INVESTIGATIONS}

MRI showed a fistula tract, thickened small bowel loops with a small quantity of fluid in the pelvis (figure 1). The MRI report suggested CD. A barium follow-through showed a dilated distal ileum and narrowing in multiple parts of the colon. Colonoscopy showed significant inflammation in the ascending colon. Biopsies were sent for histology but not for culture. Histology showed noncaseating granulomata, with no acid fast bacilli (AFB), consistent with $C D$ but a differential of TB could not be ruled out. A CT scan showed some subcentimetre intra-abdominal lymph nodes, not amenable to percutaneous biopsy. A chest $\mathrm{x}$-ray was clear.

\section{DIFFERENTIAL DIAGNOSIS}

$\mathrm{TB}$ and $\mathrm{CD}$ were the principle differential diagnoses. In the absence of any mycobacterial growth or evidence of TB on histology, a provisional diagnosis of CD was made.

\section{TREATMENT}

Treatment for $\mathrm{CD}$ was started with prednisolone while a seton suture was inserted in the persistent peri-anal fistula. The patient did not improve and re-presented with an ischio-rectal abscess on the opposite side. This was incised, tissue was sent for histology and swabs were sent for culture. The histology showed florid granulomatous inflammation. A second colonoscopy was performed to assess the need for an antitumour necrosis factor (TNF) $\alpha$ agent, namely adalimumab. Colonoscopy showed continuing active inflammation. No further samples were taken for histology or culture. Before commencing adalimumab a tuberculin skin test (TST) showed a $16 \mathrm{~mm}$ response and a Quantiferon Gold IT interferon $\gamma$ release assay (IGRA) was negative. Within 1 month of starting adalimumab 


\section{BMJ Case Reports}

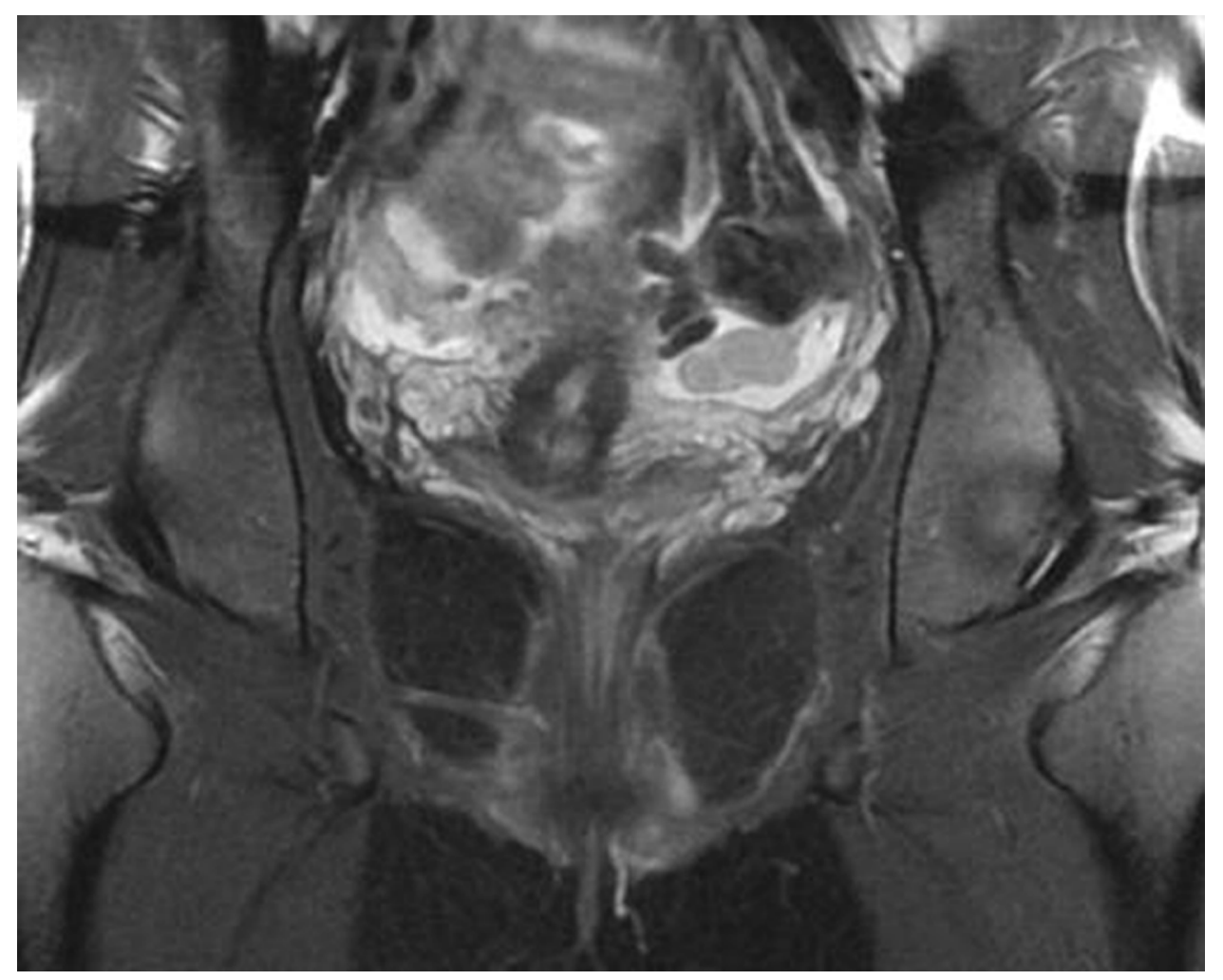

Figure 1 Initial MRI showing fistula tract and thickened small bowel loops with fluid in pelvis.

she presented with fever, rigors, diarrhoea and lethargy. A CT scan showed no abscesses, but found intra-abdominal fluid and multiple enlarged mesenteric lymph nodes. Adalimumab was immediately stopped. Her fever continued despite intravenous tazocin. Ascitic fluid was sent for culture (including for AFB) and all her previous samples were re-evaluated. At this point, an isolated acid fast bacillus was discovered on re-sectioning her first colonoscopic biopsy taken 8 months previously. She was commenced on quadruple therapy for TB (rifampicin, isoniazid, pyrazinamide and ethambutol).

\section{OUTCOME AND FOLLOW-UP}

On commencement of anti-TB therapy, she improved dramatically. Fully sensitive Mycobacterium tuberculosis subsequently grew from ascitic fluid. An HIV test was negative. She was discharged from hospital 1 week later and is currently well on treatment.

\section{DISCUSSION}

The phenomenon of mycobacterial infection revealed by the use of anti-TNF $\alpha$ agents is well recognised. ${ }^{3}$ Guidance exists on assessment for latent or active TB infection before the use of such agents. ${ }^{4}$ When screening for latent TB, a chest $x$-ray and TST or IGRA are performed. Our patient had a normal chest $\mathrm{x}$-ray and a positive TST. The latter could have been due to either mycobacterial exposure or prior BCG. If this had been interpreted as a marker of latent TB infection one possible option might have been to prescribe isoniazid chemoprophylaxis and continue with anti-TNF $\alpha$ therapy. A risk from this course of action arises when active $T B$, rather than latent $T B$, is present, isoniazid monotherapy is insufficient to treat the higher mycobacterial burdens of active TB and will eventually select for isoniazid resistance, although a false sense of clinical response may occur initially as isoniazid kills sensitive organisms. Our patient had an IGRA Quantiferon Gold IT, in addition to a TST. BCG would not have affected an IGRA, given the specificity of this test for $M$ tuberculosis infection. Her positive TST with a negative IGRA could have been interpreted as a false positive TST due to her prior BCG, but this was in fact a false negative IGRA.

Our patient gave no clear exposure history to $\mathrm{TB}$, had a clear CXR and had had BCG as a child. Although taking a BCG vaccination history is routine, its value in reducing the probability of a TB diagnosis is uncertain. The efficacy of BCG varies between populations, may wane with time and some argue may apply more to reactivation of latent infection than new exposure to TB. ${ }^{7}$ In some patients for example, with established rheumatoid arthritis, there may be no additional clinical considerations before starting anti-TNF. However in our patient there was the added complexity that active TB was a possibility.

The UK NICE guidelines do not advocate the use of IGRAs or tuberculin skin tests for the diagnosis of active TB. ${ }^{8}$ These tests do not have sufficient sensitivity to exclude disease when results are negative and lack the specificity to distinguish latent from active disease when results are positive. ${ }^{9}$ The diagnosis of active TB depends on clinical suspicion and sampling for culture. The clinical pictures of intestinal TB and CD can be very similar, although some differences have been reported. ${ }^{10}$ At colonoscopy and on histology TB can cause superficial, transverse ulcers which do not penetrate beyond the muscularis mucosa. In CD, ulcers can be longitudinal and serpiginous. Granulomas in 
the intestine or local lymph nodes can be non-caseating with either diagnosis, but only caseating with $\mathrm{TB}$ and some other infections. Mycobacterial culture of suitable samples (lymph node, intestinal tissue, fluid or pus) not placed in formalin is key to the diagnosis of TB. Swabs are not suitable for mycobacterial culture. Staining for AFB may occasionally be misleading as atypical mycobacteria may be found in the intestine. In coming years new PCR methodologies will augment diagnostic rates. ${ }^{11}$

The diagnosis of active TB depends on clinical suspicion and sampling for culture. The clinical pictures of intestinal $\mathrm{TB}$ and $\mathrm{CD}$ can be very similar, although some differences have been reported. ${ }^{10}$

Clinical suspicion is influenced by epidemiological knowledge. CD is uncommon in Saudi Arabia with a reported incidence of 0.94 per 100000 , compared with 8.3 per 100000 in the UK. ${ }^{12}{ }^{13}$ The prevalence of TB in Saudi Arabia is 22 per 100000 and not that much higher than the overall prevalence in the UK. ${ }^{1}$ However, in the UK in 2009 the prevalence of all forms of TB ranged from 7 per 100 000 (only a fraction of which would be intestinal TB) in Caucasians to 273 per 100000 in Black Africans. ${ }^{2}$

\section{Learning points}

- The above case highlights a number of issues that may confront a clinician in the UK.

- TB should be a differential diagnosis for CD in patients from areas of higher TB endemicity.

- It is difficult to distinguish TB from CD on clinical grounds.

- Samples, not placed in formalin, should be sent for 'AFB' culture.

- Care should be taken in interpreting IGRAs and the TST.

- Following deterioration on anti-TNF treatment, TB should be suspected regardless of previous investigations.

\section{Competing interests None.}

Patient consent Obtained.

\section{REFERENCES}

1. World Health Organisation. http://www.emro.who.int/stb/pdf/CountryProfilesaa-08.pdf (accessed 11 November 2011).

2. Health Protection Agency. http://www.hpa.org.uk/web/ HPAwebgHPAwebStandard/HPAweb_C/1195733758290, (accessed 11 November 2011)

3. Keane J, Gershon S, Wise RP, et al. Tuberculosis associated with infliximab, a tumor necrosis factor alpha-neutralizing agent. N Engl J Med 2001;345:1098-104.

4. British Thoracic Society Standards of Care Committee. BTS recommendations for assessing risk and for managing Mycobacterium tuberculosis infection and disease in patients due to start anti-TNF- treatment. Thorax 2005;60:800-5.

5. Ding T, Ledingham J, Luqmani R, et al.; Standards, Audit and Guidelines Working Group of BSR Clinical Affairs Committee; BHPR. BSR and BHPR rheumatoid arthritis guidelines on safety of anti-TNF therapies. Rheumatology (Oxford) 2010;49:2217-9.

6. Sterne JA, Rodrigues LC, Guedes IN. Does the efficacy of BCG decline with time since vaccination? Int J Tuberc Lung Dis 1998;2:200-7.

7. Smith D, Wiegeshaus E, Balasubramanian V. An analysis of some hypotheses related to the Chingelput bacille Calmette-Guérin trial. Clin Infect Dis 2000;31 (Suppl 3):S77-80.

8. Tuberculosis. Clinical diagnosis and management of tuberculosis, and measures for its prevention and control. NICE Guideline March 2011. http:// www.nice.org.uk/guidance/CG117 (accessed 12 January 2012).

9. Mazurek GH, Jereb J, Vernon A, et al.; IGRA Expert Committee; Centers for Disease Control and Prevention (CDC). Updated guidelines for using Interferon Gamma Release Assays to detect Mycobacterium tuberculosis infection United States, 2010. MMWR Recomm Rep 2010;59:1-25.

10. Sharma MP, Bhatia V. Abdominal tuberculosis. Indian J Med Res 2004;120:305-15.

11. Boehme CC, Nabeta P, Hillemann D, et al. Rapid molecular detection of tuberculosis and rifampin resistance. N Engl J Med 2010;363:1005-15.

12. Al-Ghamdi AS, Al-Mofleh IA, Al-Rashed RS, et al. Epidemiology and outcome of Crohns disease in a teaching hospital in Riyadh. World J Gastroenterol 2004:10:1341-4.

13. NHS Clinical Knowledge Summaries - Crohns disease. http://www.cks. nhs.uk/crohns disease/background information/incidence and prevalence (accessed 12 January 2012).

This pdf has been created automatically from the final edited text and images.

Copyright 2012 BMJ Publishing Group. All rights reserved. For permission to reuse any of this content visit http://group.bmj.com/group/rights-licensing/permissions.

BMJ Case Report Fellows may re-use this article for personal use and teaching without any further permission.

Please cite this article as follows (you will need to access the article online to obtain the date of publication).

Maitra RK, Bowling T, Venkatesan P, Maxwell-Armstrong C. Crohn's disease or TB - the perennial question and diagnostic pitfalls. BMJ Case Reports 2012;10.1136/bcr.01.2012.5620, Published XXX

Become a Fellow of BMJ Case Reports today and you can:

- Submit as many cases as you like

- Enjoy fast sympathetic peer review and rapid publication of accepted articles

- Access all the published articles

- Re-use any of the published material for personal use and teaching without further permission

For information on Institutional Fellowships contact consortiasales@bmjgroup.com

Visit casereports.bmj.com for more articles like this and to become a Fellow

Keep up to date with all published cases by signing up for an alert (all we need is your email address) http://casereports.bmj.com/cgi/alerts/etoc 